\title{
Can older adults enhance task-switching performance by verbal self-instructions?The influence of working-memory load and early learning
}

\author{
Jutta Kray ${ }^{*}$, Joanna Lucenet ${ }^{2}$ and Agnès Blaye ${ }^{2}$ \\ ' Development of Language, Learning, and Action, Department of Psychology, Saarland University, Saarbrücken, Germany \\ 2 Laboratoire de Psychologie Cognitive, UMR 6146, University de Provence, Marseille, France
}

\author{
Edited by: \\ Arthur F. Kramer, University of Illinois at \\ Urbana-Champaign, USA \\ Reviewed by: \\ Yaakov Stern, Columbia University, \\ USA \\ Chandramallika Basak, Rice University, \\ USA \\ Ulrich Mayr, University of Oregon, USA \\ ${ }^{*}$ Correspondence: \\ Jutta Kray, Development of Language, \\ Learning and Action, Department of \\ Psychology, Saarland University, P.O \\ Box 1511 50, D-66041 Saarbrücken \\ Germany. \\ e-mail:j.kray@mx.uni-saarland.de
}

In this study we examined (a) whether verbal self-instructions can enhance task-switching performance in younger and older adults, and (b) whether verbal self-instruction benefits on task switching are smaller when memory demands on keeping track of the task sequence are reduced by spatial task cueing. Task-switching ability was measured as the difference in performance between single-task and mixed-task blocks (termed mixing costs), in which participants switched between two tasks A and B. One group of participants performed the switching tasks with spatial task cues, indicating which of the two tasks has to be performed, thereby reducing demands on the endogenous control of serial task order (low task-sequencing load). The other group switched between tasks without external task cues (high task-sequencing load). To investigate the influence of verbal self-instructions on task switching, participants either named aloud the next task during task preparation (task-naming condition) or they did not verbalize (control condition). Results indicated that age differences in verbalization benefits on mixing costs depend on early learning whereby benefits were generally larger when subjects had some prior practice in task switching alone, and that verbalization benefits did not differ between the two task-sequencing load conditions. These findings suggest that task naming is a suitable cognitive intervention for enhancing the control of task switching in younger and older adults, even if memory load is reduced, and that for the efficient application of this strategy it first has to be coordinated with task switching, which is easier when task switching is already practiced.

Keywords: aging, task switching, verbalization benefits, working-memory load, task-sequencing function, endogenous task control

\section{INTRODUCTION}

It is now a well documented finding that older adults have the greatest impairments in cognitive tasks relying on cognitive control or executive control processes primarily in those associated with the coordination, maintenance, and monitoring processes required in dual-task or global task-switching situations (for a review, see Kramer and Kray, 2006; for a meta-analysis, see Verhaeghen and Cerella, 2002). To date there is already some evidence that older adults can enhance cognitive control deficits by cognitive interventions such as practice and training (e.g., Bherer et al., 2005; Basak et al., 2008; Dahlin et al., 2008; Karbach and Kray, 2009), suggesting a substantial amount of cognitive plasticity at the behavioral and neuronal level (Lindenberger et al., 2006; Hertzog et al., 2009). The primary interest of the present study is to examine whether instructions to use language (i.e., the use of verbal self-instructions) is also a suitable cognitive intervention to reduce impairments in cognitive control in the elderly.

To investigate age-related differences in cognitive control or executive control in recent years, a number of studies applied the so-called task-switching paradigm (for a review, see Kramer and Kray, 2006; for a meta-analysis, see Verhaeghen and Cerella, 2002). In this type of paradigm, participants are usually instructed to perform two or more simple categorization tasks, such as classifying the value of digits as odd or even (task A) and the color of digits as red or green (task B). In single-task blocks, they have to perform only one of the two tasks (task A or B), and in mixed-task blocks, they have to switch between both tasks A and B. This design allows calculating two types of task-switching costs: Mixing costs (or general/global switch costs) are defined as the difference in performance between single- and mixed-task blocks, and are assumed to measure the general switching ability, that is, maintaining two task sets and selecting between them. Switching costs (or specific/local switch costs) are defined as the difference in performance between switch trials (i.e., switching from A to B or B to A) and non-switch trials (repeating task A or B) within mixed-task blocks (cf. Rogers and Monsell, 1995). Switching costs are supposed to measure the ability to switch tasks at trial-to-trial transitions, that is, at a more local level of switching. Most of the aging studies so far have shown that age differences are much more pronounced for mixing costs than for switching costs (Kray and Lindenberger, 2000; Cepeda et al., 2001; Mayr, 2001; Kray et al., 2004, 2008; Reimers and Maylor, 2005; Kray, 2006; Goffaux et al., 2008), suggesting that older adults primarily have deficits with the task-set maintenance and selection, and less with the switching process itself. 
There is some evidence from the cognitive neurosciences showing both types of task-switching costs are associated with the recruitment of different brain regions. For instance, Braver et al. (2003) found that the magnitude of mixing costs was selectively correlated with activity in the right anterior prefrontal cortex, and that the magnitude of switching costs was selectively correlated with activity in the left lateral parietal cortex. Moreover, there is a growing body of evidence from the aging neurosciences that neuroanatomical and neurochemical degenerations in older age are much more pronounced in the prefrontal cortex than in other parts of the brain (for reviews, see West, 1996; Raz, 2000). The prefrontal cortex is thought to be important for the efficiency of cognitive control behavior, for instance, for the active maintenance of task representations, which is especially needed in situations of task uncertainty such as low environmental prompts to behavior (e.g., Duncan, 1995; Miller and Cohen, 2001). Note that age differences in mixing costs are also larger when demands on maintaining task representations are high (for reviews, see Verhaeghen and Cerella, 2002; Kramer and Kray, 2006), for instance, when external task cues are missing (e.g., Kramer et al., 1999; Kray et al., 2002) or when the stimuli are highly ambiguous meaning that parts of the task representations are overlapping (cf. Mayr, 2001). In line with these findings, evidence from a recent neuroimaging study further supports the view that older adults have deficits in task-goal maintenance that seems to be associated with reduced recruitment in the lateral prefrontal cortex as compared to younger adults (Paxton et al., 2006). Moreover, results of this study showed that older adults, in contrast to young adults, seem to be less engaged in advance preparation and focus more on target processing when ambiguous stimuli induce task uncertainty.

In recent years, a number of studies have examined the role of language, and in particular of inner speech processes, for the efficiency to switch back and forth between simple tasks (Baddeley et al., 2001; Emerson and Miyake, 2003; Miyake et al., 2004; Saeki and Saito, 2004; Bryck and Mayr, 2005). All of these studies have found that mixing costs were substantially increased when inner speech was disrupted by articulatory suppression. Under articulatory suppression conditions, subjects had to repeat aloud an over-learned sequence of words during task preparation, such as naming the days of the week. Importantly, mixing costs were largest when task-set cues were absent and stimuli were ambiguous so that subjects needed to rely on internal cueing (e.g., Baddeley et al., 2001; Emerson and Miyake, 2003). Thus, with the presence of external task cues, the recruitment of inner speech processes was less needed in order to appropriately represent and maintain currently relevant task goals. These findings generally suggest that inner speech facilitates the activation and retrieval of task representations, thereby serving as a useful self-cueing device primarily in the absence of external cueing (cf. Emerson and Miyake, 2003). Most of the studies reported that mixing costs but not switching costs were greater under articulatory suppression, supporting the view that verbal processes influence the maintenance and selection of task goals and less the switching process itself (e.g., Emerson and Miyake, 2003; Saeki and Saito, 2004). Moreover, Bryck and Mayr (2005) found that the articulatory suppression effect was larger for high task-sequencing load conditions under which participants had to keep track of the task sequence during task switching. They argued that the function of language or verbal self-cueing during task switching is more specific namely subserving the serial order of tasks in the absence of external task cues. Hence, verbal cueing has a specific sequencing function during task switching.

In a more recent study we were not only interested in whether the disruption of inner speech or verbalizations hindered the efficient switching between tasks but also in whether the use of verbal self-instructions (naming the next task goal) enhanced task-switching performance (Kray et al., 2008). To examine this, we compared conditions in which subjects had to repeat aloud task-irrelevant words (e.g., the) during task preparation with conditions in which no verbalization was required and a condition in which subjects had to name aloud the upcoming task label (e.g., color). Similar to the previous findings, we found that mixing costs were larger when subjects had to verbalize task-irrelevant words (Baddeley et al., 2001; Emerson and Miyake, 2003; Miyake et al., 2004; Saeki and Saito, 2004; Bryck and Mayr, 2005). Moreover, mixing costs were substantially reduced when subjects used verbal self-instructions, suggesting that verbal processes have an important function for the regulation of task-switching behavior. Even more important for the present study, results of this study indicated that children and older adults showed a larger reduction of mixing costs under task-naming conditions relative to younger adults, suggesting that the use of verbal processes is quite useful for enhancing the ability to maintain and select task sets in childhood and old age.

The primary goal of the present study was to determine whether benefits of verbal cueing on mixing costs in younger and older adults vary with memory demands to keep track of the task sequence, that is, under high and low task-sequencing conditions. In order to examine age differences in task switching we used a similar version of the switching paradigm, as in our previous study (cf. Kray et al., 2008). Subjects had to perform two different types of blocks. In singletask blocks, they only performed one of two tasks (A or B) and in mixed-task blocks (A and B) they had to switch tasks on every second trial. We investigated the influence of verbal self-instructions by comparing conditions in which subjects named the next task aloud during task preparation (task-naming condition) with conditions in which no additional naming was required (control condition). As a replication of previous findings, we expected that mixing costs will be smaller and age differences will be reduced under task-naming conditions (cf. Kray et al., 2008).

A number of recent studies also suggested that verbal processes supporting the retrieval of the next task goal are primarily required when external task cues are missing (Baddeley et al., 2001; Emerson and Miyake, 2003; Miyake et al., 2004; Saeki and Saito, 2004; Bryck and Mayr, 2005). Thus, under these conditions verbal processes serve as a useful retrieval aid. In addition to replicating previous findings, a specific aim of the present study was to examine whether older adults also benefit from verbal self-instructions when demands on keeping track of the task sequence are reduced. To manipulate task-sequence memory demands, one group of participants performed the switching tasks without external cues (cf. Kray and Lindenberger, 2000; Bryck and Mayr, 2005). All targets appeared in one grid and participants only knew by the predictability of the task sequence (AABBAA and so on), which task they should perform in the next trial (see Figure 1). For the other group 


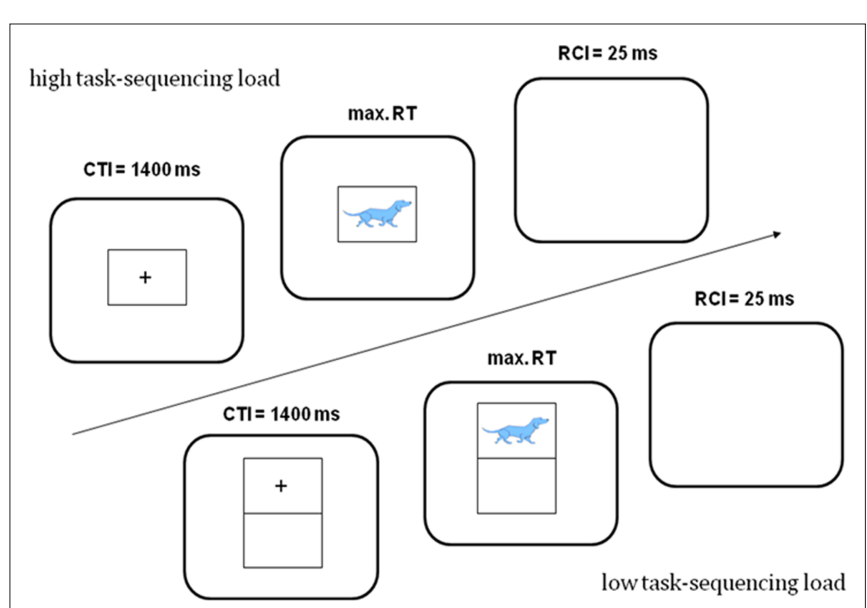

FIGURE $1 \mid \mathrm{CTI}$ = time between fixation onset and target presentation; $\mathrm{RCl}=$ time between the response and the presentation of the next fixation cross. The task sequence was AABBAA... in the high- and low task-sequencing condition. In the low task-sequencing condition the upper position indicated that task $A$ (the object task: car or dog?) was required and the lower position that task $B$ (the color task: blue or orange?) should be performed. In the verbalization conditions, task naming starts with the onset of the fixation cross.

of participants, the targets appeared in two grids (cf. Bryck and Mayr, 2005), in the upper grid subjects were instructed to perform task A and in the lower grid they should perform task B. That is, the first two targets always appeared in the upper grid, indicating task A, and the next two targets in the lower grid, indicating task B and so forth. Thus, demands on keeping track of the task sequence were lower as the spatial position of target appearance was a valid cue for each of the two tasks. In line with previous findings (Bryck and Mayr, 2005), we expected that with the presence of spatial task cues (i.e., lower task-sequencing demands), verbal cueing is less needed so that benefits of verbal self-instructions on mixing costs will be reduced, at least for younger adults. However, so far we do not know and had no specific prediction about whether older adults will give up a verbal retrieval aid in situations in which external retrieval aids can be used.

\section{MATERIALS AND METHODS PARTICIPANTS}

Results for 40 younger adults (mean age $=20.9$ years; age range $=17-30$ years; $70 \%$ female) and 45 older adults (mean age $=72.7$ years; age range $=68-80$ years; $67 \%$ female) will be reported. Participants were recruited through a database of subjects who had interest in participating in research studies at Saarland University and they were paid $7.50 € / \mathrm{h}$. Informed consent was obtained from all participants, using a procedure approved by the Internal Review Board of Saarland University.

Subjects in the two task-switching versions with a high or load task-sequence memory load (1-grid vs. 2-grids) did not differ in speed of processing $(p=0.30)$, and working-memory span $(p=0.18)$ (for a description, see the next section). We only obtained reliable age differences in the speed test, $F(1,81)=84.20, p<0.001$, $\eta^{2}=0.51$, indicating that younger adults $(M=53 ; \mathrm{SD}=6.8)$ reached a higher score than older adults $(M=38.4$; $\mathrm{SD}=7.6)$. Moreover, younger adults $(M=9.15 ; \mathrm{SD}=1.9)$ also reached a higher score on the working-memory test relative to older adults $(M=6.3$; $\mathrm{SD}=2.3), F(1,81)=38.83, p<0.001, \eta^{2}=0.32$.

\section{APPARATUS, STIMULI, AND TASKS}

We administered two psychometric tests to control whether subjects in the two task-switching versions with a high or load task-sequence memory load (1-grid vs. 2-grids) did not differ in speed of processing and working-memory span (see Participants). To measure speed of processing, participants performed a colornaming test in which they saw a sheet of several unfilled shapes (circles, crosses, triangles, squares). A template, presented on the top of the sheet, contained four colored shapes. The subject's task was to name as quickly and accurately as possible what color each test shape was on the template. The score was the number of correctly named colors within a time window of $45 \mathrm{~s}$. In the workingmemory span test, participants had to repeat aloud a sequence of digits ranging from 2 to 8 in the reversed order of presentation (adapted from Wechsler, 1981,2003). Two items for each span were given and the score was the number of items that were correctly repeated in the reversed order.

For the switching experiment, we used IBM compatible laptops (Dell $^{\mathrm{TM}}$ Latitude $^{\mathrm{TM}}$ D820) for data collection. The stimuli were presented on the center of a WXGA 15.4-inch color monitor with a white background. Responses were registered with the q-key and the p-key of the laptop keyboard, and the experiment was programmed with the Software package E-Prime 1.1. Visual stimuli consisted of 18 pictures of three different dogs and cars and each image was presented in three different shades of orange or blue. Participants were instructed to perform two different tasks, an "object" (task A) and a "color" task (task B). In the object task, participants respond to the appearance of a dog or a car, and subjects pressed either the left (q-key) or the right (p-key) key with the left or right index finger, respectively. In the color task, they decided whether the picture was in orange or blue, and they also pressed either the left (q-key) or the right ( $\mathrm{p}$-key) key with the left or right index finger, respectively. In the half of the blocks, participants were instructed to perform the verbalization as a secondary task, that is, they were instructed to name the next task to be performed. For instance, they said "picture" or "color" prior to target presentation and they started verbalizing with the onset of the fixation cross in each trial.

\section{DESIGN}

Task-type block (single vs. mixed) and verbalization (task naming vs. control condition) was manipulated within subjects. In singletask blocks, participants either performed the "object" or "color" task, and in mixed-task blocks, they had to switch between both tasks on every second trial. In the task-naming condition, subjects named the next task at the onset of the fixation cross. Blocks of task naming and blocks without task naming were grouped together and the sequence of verbalization was counterbalanced across subjects to control for order effects. Task-sequencing load (high vs. low) was manipulated between subjects. In the high task-sequencing load condition, all stimuli were presented in one grid so that demands on keeping track of the task sequence were high. In the low tasksequencing load condition, the stimuli were presented in one of two grids (see Figure 1). In sum, we used a 2 (task type) $\times 2$ 
(verbalization) $\times 2$ (task-sequencing load) design and controlled for order effects of performing the task-naming condition or the control condition first.

\section{PROCEDURE}

Participants were tested in a one-session experiment. At the beginning of each session the subjects received a short demographic questionnaire. Then, they performed two psychometric tests, the color-naming test, and a working-memory span test (see Participants), followed by the switching task.

The switching task consisted of an introduction phase and a testing phase. In the introduction phase, participants performed first two single-task blocks (task A and task B in isolation) followed by two mixed-task blocks. The testing phase consisted of eight singletask and eight mixed-task blocks. Two single-task blocks (task A and task B) and two mixed-task blocks were always grouped together. Half of the participants, first performed the introduction phase and testing phase without verbalization, and then with verbalization (control first), and the other half of the participants first performed all blocks with verbalization, and then without verbalization (task naming first). The procedure for the phase with verbalization was identical, except that subjects were additionally instructed to start the verbalization at the onset of the fixation cross. Each block in the practice phase and the testing phase consisted of 17 trials whereas the first trial in each block was not analyzed. Both single- and mixed-task blocks consisted of an equal number of four stimulus types (dogs/orange, dogs/blue, cars/orange, cars/blue). In addition, mixed-task blocks consisted of an equal number of non-switch and switch trials.

Experimental trials started with the presentation of a fixation cross that was centrally presented for $1,400 \mathrm{~ms}$ prior to target presentation (see Figure 1). Then, the target appeared and was presented until a response was made. The time interval between the response and the next fixation cross was $25 \mathrm{~ms}$. Before each of the experimental blocks, an instruction window appeared indicating whether task A or task B, or both tasks had to be performed and whether naming of the next task was required in addition. After each block, participants received feedback about their mean response time and percentage of errors. After half of the blocks, subjects had a short break of 5-10 min.

\section{RESULTS}

\section{DATA ANALYSIS}

The first trial in each block was excluded from data analysis. Furthermore, latencies faster than $180 \mathrm{~ms}$ and slower than $3000 \mathrm{~ms}$ were excluded from the analysis $(0.1 \%$ for the younger adults; and $0.6 \%$ for the older adults). Analyses of latencies were based on correct trials and on mean reaction time (RT). Because differences between logarithms are equivalent to ratio scores and therefore less sensitive to age differences in baseline conditions, we also performed ANOVAs based on log-transformed RT (cf. Kray and Lindenberger, 2000).

\section{SEQUENCE EFFECTS OF VERBALIZATION}

We first conducted an overall ANOVA to control for order effects of starting with the task-naming condition or with the control condition. The ANOVA included the between-subjects factors Age group,
Task-sequencing group, and Verbalization order (control first, task-naming first) and the within-subjects factors, Block type (single, mixed) and Verbalization (task naming, control). The ANOVA on latencies revealed main effects of Block type, $F(1,77)=44.69$, $p<0.001, \eta^{2}=0.37$, and Verbalization, $F(1,77)=32.67, p<0.001$, $\eta^{2}=0.30$, as well as a number of reliable higher-order interactions with the factor Verbalization order: Verbalization $\times$ Verbalization order: $F(1,77)=35.85, p<0.001, \eta^{2}=0.32$; Block type $\times$ Verbaliz ation $\times$ Verbalization order: $F(1,77)=21.65, p<0.001, \eta^{2}=0.22$; Age group $\times$ Block type $\times$ Verbalization $\times$ Verbalization order: $F(1,77)=7.85, p=0.01, \eta^{2}=0.09$. The ANOVA on error rates also showed main effects of Block type, $F(1,77)=171.75, p<0.001$, $\eta^{2}=0.69$, and Verbalization, $F(1,77)=35.16, p<0.001, \eta^{2}=0.34$, as well as higher-order interactions with Verbalization order: Verbalization $\times$ Verbalization order: $F(1,77)=4.60, p<0.05$, $\eta^{2}=0.06$; Age group $\times$ Verbalization $\times$ Verbalization order: $F(1,77)=6.95, p=0.01, \eta^{2}=0.08$; Age group $\times$ Block type $\times$ Verb alization $\times$ Verbalization order: $F(1,77)=4.96, p<0.05, \eta^{2}=0.06$. To understand the nature of these interactions, we conducted separate ANOVAs for the two verbalization order conditions.

\section{Starting with the control condition}

The ANOVA included the between-subjects factors Age group and Task-sequencing group and the within-subjects factors, Block type and Verbalization. All effects remained reliable on log-transformed RT, unless reported otherwise. Latencies of all experimental conditions are reported in Table $\mathbf{1}$ and mixing costs are displayed in Figure 2A as a function of age group, task-sequencing group, and verbalization condition.

The results on latencies revealed reliable verbalization benefits, $F(1,38)=53.83, p<0.001, \eta^{2}=0.59$, that is, faster latencies under task-naming conditions than under control conditions. Verbalizations benefits were larger in mixed-task blocks than in single-task blocks, $F(1,38)=34.7, p<0.001, \eta^{2}=0.48$, indicating that mixing costs were largely reduced under task-naming conditions. Moreover, the reduction of mixing costs under task naming was more pronounced for older adults than for younger adults, $F(1,38)=9.14, p<0.01, \eta^{2}=0.19$ (see Figure 2A). Post hoc comparisons showed that age differences in mixing costs were only reliable for the control condition, $F(1,40)=10.72, p<0.01, \eta^{2}=0.21$, but disappeared for the task-naming condition $(p=0.31)$. However, we found no reliable interactions with task-sequencing load and variables of interest.

The results on error rates indicated larger error rates in mixedthan single-task blocks, that is, reliable mixing costs, $F(1,38)=24.20$, $p<0.001, \eta^{2}=0.40$, and error rate were also larger in verbalization than control blocks, $F(1,38)=6.88, p=0.01, \eta^{2}=0.15$. Both effects did not interact with Age group or Task-sequencing load group.

\section{Starting with the task-naming condition}

The ANOVA was identical to the one described in the previous section. Latencies of all experimental conditions are reported in Table 2 and mixing costs are displayed in Figure $2 \mathbf{B}$ again as a function of age group, task-sequencing load group, and verbalization condition.

Results of mean latencies revealed age differences in mixing costs, $F(1,39)=10.93, p<0.01, \eta^{2}=0.22$, that interacted with the task-sequencing load condition, $F(1,39)=4.80, p<0.05, \eta^{2}=0.11$ 
Table 1 | Mean (SE) of Latencies (ms) as a Function of Block Type, Age Group, Task-sequencing Load, and Verbalization Condition for the Control Condition First Group.

\begin{tabular}{|c|c|c|c|}
\hline & \multicolumn{3}{|c|}{ High task-sequencing load (1 Grid) } \\
\hline & Task naming & Control & Verbalization benefits \\
\hline \multicolumn{4}{|l|}{ YOUNG } \\
\hline Single blocks & $399(21)$ & $431(28)$ & $-32(18)$ \\
\hline Mixed blocks & $463(29)$ & $591(48)$ & $-128(27)$ \\
\hline Mixing costs & $64(13)$ & $161(27)$ & $-97(22)$ \\
\hline \multicolumn{4}{|l|}{ OLD } \\
\hline Single blocks & 594 (39) & $580(33)$ & $14(20)$ \\
\hline Mixed blocks & $648(41)$ & $929(75)$ & $-281(46)$ \\
\hline \multirow[t]{3}{*}{ Mixing costs } & $54(23)$ & $349(53)$ & $-294(55)$ \\
\hline & \multicolumn{3}{|c|}{ Low task-sequencing load (2 Grids) } \\
\hline & Task naming & Control & Verbalization benefits \\
\hline \multicolumn{4}{|l|}{ YOUNG } \\
\hline Single blocks & $431(23)$ & $478(24)$ & $-47(17)$ \\
\hline Mixed blocks & $488(30)$ & $600(38)$ & $-112(28)$ \\
\hline Mixing costs & $57(14)$ & $122(28)$ & $-65(27)$ \\
\hline \multicolumn{4}{|l|}{ OLD } \\
\hline Single blocks & $661(65)$ & $645(48)$ & $16(55)$ \\
\hline Mixed blocks & $769(83)$ & 962 (105) & $-193(52)$ \\
\hline Mixing costs & $109(30)$ & 317 (88) & -209 (82) \\
\hline
\end{tabular}

Mixing costs = RT mixed blocks $-R T$ single blocks; Verbalization benefits $=R T$ task naming - RT control condition.

(see Figure 2B). Age differences in mixing costs were reliable only for conditions with a low task sequencing load, $F(1,20)=15.84$, $p<0.01, \eta^{2}=0.44$, but not for a high task sequencing load $(p=0.45)$. Post hoc comparisons showed that mixing costs were larger under high task-sequencing conditions only for the younger age group, $F(1,18)=9.67, p<0.01, \eta^{2}=0.35$, but not for the older age group $(p=0.20)$. However, we found no verbalization benefits on mixing costs when subjects did the task-naming condition first. Therefore, we run control analyses that will be reported in the next section.

The ANOVA results on error rates indicated reliable mixing costs, $F(1,38)=20.45, p<0.001, \eta^{2}=0.34$, that is, subjects made more errors in mixing blocks than in single-task blocks. We also found an effect of verbalization, $F(1,38)=28.91, p<0.01, \eta^{2}=0.43$, showing than subjects made more errors under task-naming conditions than under control conditions when they performed the verbalization task first, and this effect was even more pronounced for older adults than for younger adults, $F(1,38)=9.34, p<0.001, \eta^{2}=0.19$.

\section{CONTROL ANALYSES}

In line with our previous study (Kray et al., 2008), in which the subjects also received practice in task switching prior to the verbalization manipulations, we found substantial verbalization benefits on mixing costs when subjects already had some practice in task switching (control first group). However, no reliable verbalizations benefits on mixing costs were found for the group starting with the task-naming condition. To rule out that these findings are influenced by differential within-session practice effects in error rates across the two groups, we first run an additional analysis on error rates, and then we conducted the same ANOVA on latencies as before but only for the second half of the first session in which performance is more stable Furthermore, we will report data from a small control study in which we examined whether verbalization benefits on mixing costs will occur when subjects received more practice (i.e., two sessions instead of only one).

\section{Within-session practice}

Within-session practice effects were examined by comparing performance between a first and a second series of two single- and two mixed-task blocks in each verbalization condition. First, we run a control analysis on error rates, including the factor Series, in order to control whether age differences in verbalization benefits on mixing costs are influenced by changes in error rates and whether this interacts with the order effect of task naming. Results indicated that subjects made more errors on mixed than single-task blocks, $F(1,81)=64.10, p<0.001, \eta^{2}=0.36$, and on verbalization than control blocks, $F(1,81)=29,86, p<0.001, \eta^{2}=0.27$, as well as a significant practice effect, $F(1,81)=13.77, p<0.001, \eta^{2}=0.15$, suggesting that subjects made less errors in the second series than in the first. No other effect reached significance. Hence, it can be ruled out that changes in error rates can explain the pattern of findings across the two order conditions.

Second, we run a further ANOVA on latencies that only include trials from the second series of trials so that the results are less influenced by early learning. Our results revealed that all significant findings remained except one. The interaction between age group, verbalization, and trial types was no longer significant $(p=0.08)$, suggesting that younger and older adults' verbalization benefits on mixing blocks only differ relatively early in learning.

\section{Across-session practice: A control study}

To further understand the influence of early practice on using verbalizations, a subsample of our older adults sample $(n=28)$ received a second session of practice that was identical to the first. Given that the task-sequencing factor did not interact with any variable of interest, we displayed the new results in Figure 3 as a function of session (session 1, session 2), verbalization (task naming, control), and task-naming order condition (control first, tasknaming first).

Figure 3 shows that across-session practice effects were more pronounced in those conditions that subjects practiced first, as indicated by a significant interaction between block type, verbalization, session, and verbalization order, $F(1,26)=8.77, p<0.01$, $\eta^{2}=0.25$. Of most interest in this control study was that early learning strongly influenced the occurrence of verbalization benefits on mixing costs. For task-naming first group verbalization benefits on mixing costs were absent in session 1 , but were reliable in session $2, F(1,19)=12.5, p<0.01, \eta^{2}=0.40$. Also note that in session 2, the magnitude of mixing costs in the control condition and task-naming condition was about the same independently of the verbalization order. 


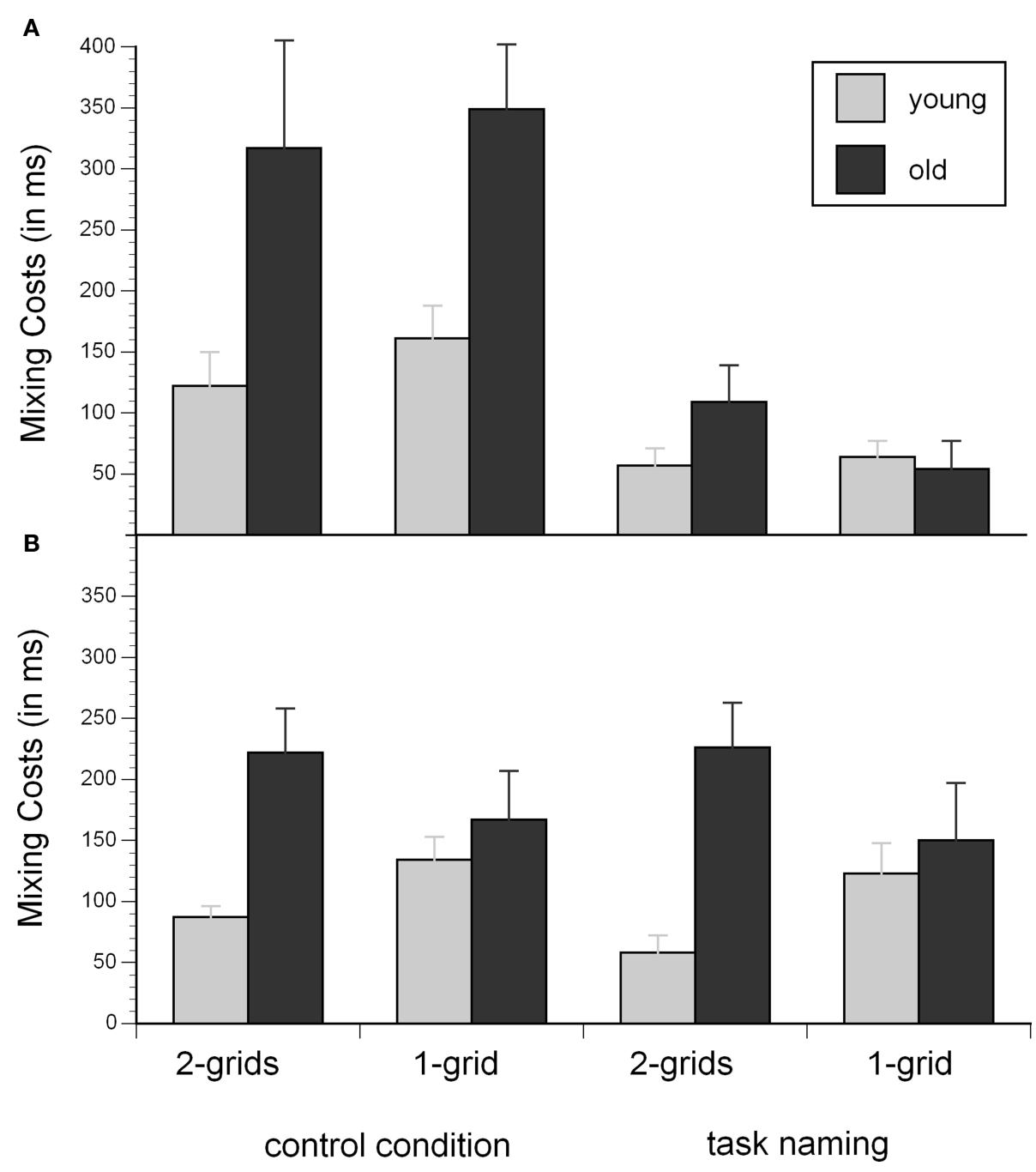

FIGURE 2 | Mixing costs are displayed as a function of age group (young/old), task-sequencing load (1-grid/2-grids), and verbalization condition (control condition/task naming) separately for the group starting with the control condition (A) and the group starting with the task-naming condition (B).

\section{DISCUSSION}

The aims of this study were to examine (a) age differences in the efficiency of task switching when verbal self-instructions (task naming) support task preparation and (b) whether verbalization benefits are larger when demands on keeping track of the task sequence are high. To investigate this, we determine younger and older adults' mixing costs under conditions with or without prior naming of the upcoming task. One group of younger and older adults did this under high task-sequencing load conditions (without spatial cueing, i.e., 1-grid version) and the other group under low task-sequencing load conditions (with spatial task cueing, i.e., 2-grids version).

At first, consistent with a previous study mixing costs were reduced under task-naming conditions (Kray et al., 2008), generally consistent with the view that language promotes task set retrieval (Baddeley et al., 2001; Emerson and Miyake, 2003; Miyake et al., 2004; Saeki and Saito, 2004; Kray et al., 2008). A new finding of this study is that benefits of task naming are dependent on early practice in task switching. It seems that task naming benefits are substantial when subjects already have some practice in task switching without additional verbalization. By contrast, task naming benefits are absent during early learning (i.e., in session 1) for the group that did the task-naming condition first, and as indicated by our control study, verbalization benefits only occur when subjects received even more practice (i.e., in session 2). This finding suggests that subjects first need to learn the coordination of applying language and switching between task sets before the naming strategy becomes beneficial.

However, the question remains why is task naming such a useful strategy although subjects have to perform a secondary task? We think that task naming strongly support task preparation in two ways. First, given that subjects were instructed to verbalize at the onset of the fixation cross (i.e., at the beginning of the preparation interval), they had an additional cue when to begin with the retrieval process, which is most efficient if 
it starts early during the preparation interval. Second, there is some evidence that subjects sometimes tend to engage in advance preparation and sometimes not, suggesting that they are not always optimally prepared for the next task (see failureto-engage account by De Jong, 2001; Nieuwenhuis and Monsell,

Table 2 | Mean (SE) of Latencies (ms) as a Function of Block Type, Age Group, Task-sequencing Load, and Verbalization Condition for the Task Naming First Group.

High task-sequencing load (1 Grid)

Task naming Control Verbalization benefits

\begin{tabular}{lllr}
\hline YOUNG & & & \\
Single blocks & $434(19)$ & $419(13)$ & $15(12)$ \\
Mixed blocks & $556(34)$ & $552(21)$ & $4(30)$ \\
Mixing costs & $123(25)$ & $134(19)$ & $-11(30)$ \\
OLD & & & \\
Single blocks & $527(39)$ & $514(34)$ & $13(14)$ \\
Mixed blocks & $684(76)$ & $686(62)$ & $-2(52)$ \\
Mixing costs & $157(52)$ & $172(44)$ & $-15(58)$ \\
\hline
\end{tabular}

Low task-sequencing load (2 Grids)

Task naming Control Verbalization benefits

\begin{tabular}{lrrr}
\hline YOUNG & & & \\
Single blocks & $403(20)$ & $396(22)$ & $7(14)$ \\
Mixed blocks & $461(24)$ & $483(24)$ & $-22(11)$ \\
Mixing costs & $58(14)$ & $87(9)$ & $-29(17)$ \\
OLD & & & \\
Single blocks & $606(50)$ & $583(29)$ & $22(36)$ \\
Mixed blocks & $831(74)$ & $805(58)$ & $26(33)$ \\
Mixing costs & $226(37)$ & $222(36)$ & $4(26)$ \\
\hline
\end{tabular}

Mixing costs $=R T$ mixed blocks $-R T$ single blocks; Verbalization benefits $=R T$ task naming - RT control condition.
2002). Thus, task naming may increase the likelihood to engage in advance preparation in most of the trials and thereby strongly reduces mixing costs.

Also consistent with our previous study older adults show larger benefits of verbalization relative to younger adults if they have already some practice in task switching (Kray et al., 2008), but this advantage disappears with practice in the present study. Moreover, in contrast to our previous findings, age differences in task switching disappear under task-naming conditions, suggesting that older adults are able to fully compensate for deficits in the regulation of task-switching behavior by making use of task-naming strategies. However, note that relative to the previous study, we used less complex stimuli and a smaller stimulus set-size, which might explain the difference in findings across studies. As there is at least some evidence that older adults fail to make less spontaneous use of inner speech processes to support action control relative to younger adults (Meichenbaum, 1974), they particularly profit if they are explicitly instructed to use them during task preparation. At first glance, our results seem to be inconsistent with a number of studies that did not find evidence for age-related differences in task preparation, suggesting that younger and older adults are more efficient in task switching, when they have more preparation time (e.g., Kramer et al., 1999; Kray and Lindenberger, 2000). As already noted we assume that the induced task naming strategy might particularly promotes the optimal timing and application of task-set retrieval in contrasts to situations in which subjects simply have more time to prepare and make use of less efficient retrieval strategies or do not use a strategy at all. This view is consistent with recent findings by Paxton et al. (2006) indicating that older adults differ from younger adults in using context (cue) information and that the use of context information can be enhanced in older adults by naming strategies and practice. Alternatively, it could be that task naming stronger activates currently relevant task-set representations so that older adults may suffer less from stimulusinduced interference so that age differences in task switching are strongly reduced.

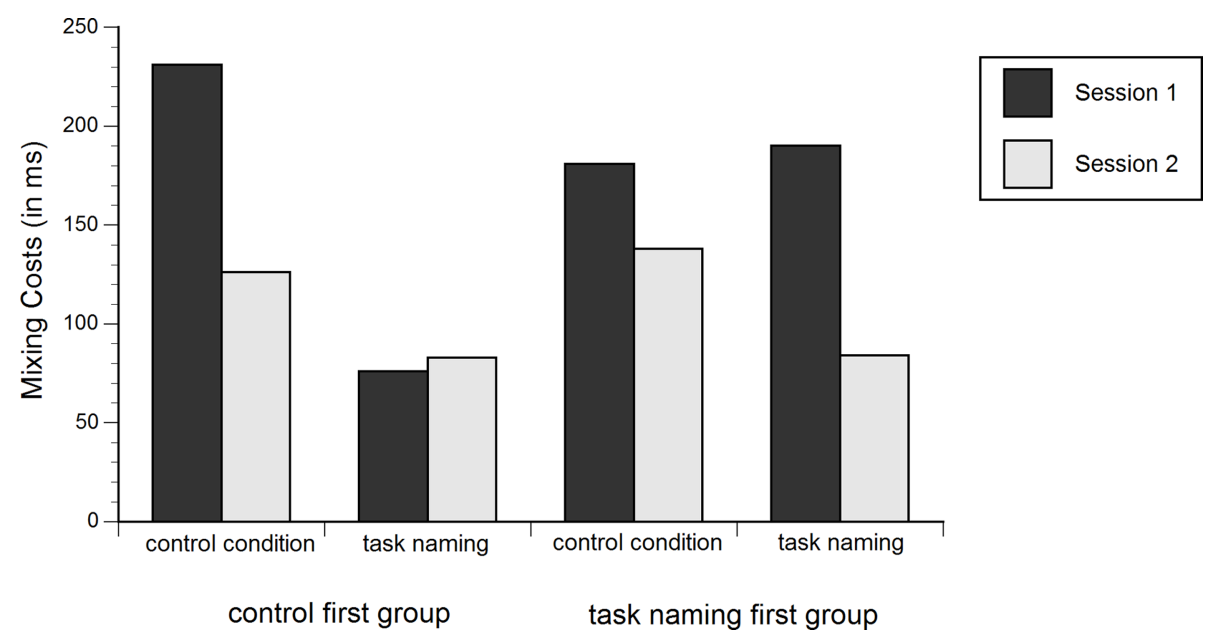

FIGURE 3 | Mixing costs are displayed as a function of session (session 1/ session 2), verbalization condition (control condition/task naming), and verbalization order (control first group/task-naming first group). 
Finally, we were specifically interested in whether age differences in using verbalizations during task switching are dependent on memory load conditions, and expected that verbalizations benefits are lower with the present of spatial task cues at least for younger adults. Given that it has been suggested that verbal processes have a specific sequencing function during task switching that support the endogenous control of serial task order in the absence of external task cues (Bryck and Mayr, 2005), we assumed that verbalization benefits should be larger for high sequencing load conditions (1-grid group) than for low sequencing load conditions (2-grids group). Our results indicated no reliable interactions between verbalization benefits, mixing costs, and task-sequencing load, neither for the younger nor for the older age group. Bryck and Mayr, in contrast, found that switching costs were sensitive to this memory load manipulation. However, in their study they used shorter time intervals between response and the next stimulus and examined the impact of language by articulatory suppression, which could explain the inconsistencies in findings. Moreover, we of course do not know whether the sequencing function becomes essential for more complex sequencing demands. Therefore, more research is needed to specify under which conditions verbal processes have a specific sequencing function during endogenous control of serial task orders.

Although we found no interaction with verbalizations, our results show an effect of task-sequencing load for the younger age group that did the verbalization condition first. This finding suggests that younger adults were more efficient in task switching when the spatial position cued the currently relevant task and there was no need to keep track of the task sequence. However, a spatial cueing effect was only found for the group with higher control demands during early learning in which the coordination of naming and switching was required. Interestingly, a tendency for an opposite effect can be seen for the older adults, namely they were less efficient under low sequencing conditions. Individual differences in working-memory between the two task-sequencing load groups could explain this finding, but this seems unlikely as we did not obtain significant differences in a separate workingmemory span test between experimental groups. Alternatively, one has to keep in mind that in the 2 -grids version, subjects additionally

\section{REFERENCES}

Baddeley, A., Chincotta, D., and Adlam, A. (2001). Working memory and the control of action: evidence from task switching. J. Exp. Psychol. Gen. 130, 641-657.

Basak, C., Boot, W. R., Voss, M. W., and Kramer,A. F. (2008). Can training in a real-time strategy video game attenuate cognitive decline in older adults? Psychol. Aging 23, 765-777.

Bherer, L., Kramer, A. F., Peterson, M. S., Colcombe, S., Erickson, K., and Becic, E. (2005). Training effects on dual-task performance: are there age-related differences in plasticity of attentional control? Psychol. Aging 20, 695-709.

Braver, T.S., Reynolds, J.R., and Donaldson, D. I. (2003). Neural mechanisms of transient and sustained cognitive

had to remember, which task to perform at which position and that also increases memory load and this seems to be particularly difficult for older adults who began with the verbalization as a secondary task.

In sum, our findings further support the view that verbal processes in terms of verbal self-instructions can be suitable retrieval aids that enhance the efficiency of task switching in younger and older adults. In addition to the replication of previous findings, the results of this study showed that the efficiency of applying verbal strategies is larger after some practice in task switching alone, suggesting that subjects need at least some practice in coordinating naming and switching. Results also provided evidence that younger and older adults use task naming as an efficient strategy even if memory demands of keeping track of the task sequence are reduced by spatial task cues, further supporting the usefulness of language processes. Finally, only younger adults made use of spatial task cueing but only when control demands were relatively high and the coordination of naming and switching was required without prior practice in switching.

Given that verbal self-instruction can be seen as such a useful cognitive intervention to enhance the control and regulation of behavior in elderly people, at least one interesting question for future aging studies would be to examine whether the use of verbal self-instructions can be trained and also be transferred to a new switching situation, supporting the general applicability of verbal cueing strategies. However, first evidence from a few intervention studies seems to suggest that the transferability of trained verbal instructions is rather limited and by this seems to be specific to the trained task-switching situation (Karbach and Kray, 2009; Karbach et al., in press).

\section{ACKNOWLEDGMENTS}

This research was funded by the German Research Foundation (DFG) thought a grant to Jutta Kray (Kr 1884/5-1) and the Agence Nationale de la Recherche (ANC) thought a grant to Agnès Blaye. Special thanks go to Julia Karbach, Noemi Hoellen, and Nicolas Chevalier for helpful comments as well as to Daniel Strass, Sandra Mang, Hannah Schmitt, and Sonja Heintz for data collection and analyzing.

Duncan, J. (1995). "Attention, intelligence, and the frontal lobes," in The Cognitive Neurosciences, ed. M. S. Gazzaniga (Cambridge, MA: MIT Press), 721-733.

Emerson, M. J., and Miyake, A. (2003). The role of inner speech in task switching: a dual-task investigation. J. Mem. Lang. 48, 148-168.

Goffaux, P., Phillips, N. A., Sinai, M., and Pushkar,D. (2008). Neurophysiological measures of task-set switching: effects of working memory and aging. $J$. Gerontol. B Psychol. Sci. Soc. Sci. 63, 57-66.

Hertzog, C., Kramer, A. F., Wilson, R. S., and Lindenberger, U. (2009). Enrichment effects on adult cognitive development. Psychol. Sci. Public Interest 9, 1-65.
Karbach, J., and Kray, J. (2009). How useful is executive control training? Age differences in near and far transfer of task-switching training. Dev. Sci. 12, 978-990.

Karbach, J., Mang, S., and Kray, J. (2010). Transfer of verbal self-instruction training in older age. Psychol. Aging 25, 677-683.

Kramer, A. F., Hahn, S., and Gopher, D. (1999). Task coordination and aging: explorations of executive control processes in the task-switching paradigm. Acta Psychol. 101, 339-378.

Kramer, A. F., and Kray, J. (2006). "Aging and divided attention," in Lifespan Cognition: Mechanisms of Change, eds E. Bialystok and F. I. M. Craik (Oxford, U.K.: Oxford University Press), 57-69. 
Kray, J. (2006). Task-set switching under cue-based and memory-based switching conditions in younger and older adults. Brain Res. 1105, 83-92.

Kray, J., Eber, J., and Karbach, J. (2008). Verbal self-instructions in task-switching: a compensatory tool for actioncontrol deficits in childhood and old age? Dev. Sci. 11, 223-236.

Kray, J., Eber, J., and Lindenberger, U. (2004). Age differences in executive functioning across the lifespan: the role of verbalization in task preparation. Acta Psychol. 115, 143-165.

Kray, J., Li, K. Z. H., and Lindenberger, U. (2002). Age-related changes in task switching components: the role of task uncertainty. Brain Cogn. 49, 363-381.

Kray, J., and Lindenberger, U. (2000). Adult age differences in task switching. Psychol. Aging 15, 126-147.

Lindenberger, U., Li, S.-C., and Bäckman, L. (2006). Delineating brain-behavior mappings across the lifespan: substantive and methodological advances in developmental neuroscience. Neurosci. Biobehav. Rev. 30, 713-717.
Mayr, U.(2001).Age differences in the selection of mental sets: the role of inhibition, stimulus ambiguity, and response set overlap. Psychol. Aging 16, 96-109.

Meichenbaum, D. H. (1974). Selfinstructional strategy training: a cognitive prosthesis for the aged. Hum. Dev. 17, 273-280.

Miller, E. K., and Cohen, J. D. (2001). An integrative theory of prefrontal cortex function. Annu. Rev. Neurosci. 24, 167-202.

Miyake, A., Emerson, M. J., Padilla, F., and Ahn, J. (2004). Inner speech as a retrieval aid for task goals: the effects of cue type and articulatory suppression in the random task cueing paradigm. Acta Psychol. 115, 123-142.

Nieuwenhuis, S., and Monsell, S. (2002). Residual costs in task switching: testing the failure-to-engage hypothesis. Psychon. Bull. Rev. 9, 86-92.

Paxton, J. L., Barch, D. M., Storandt, M., and Braver, T. S. (2006). Effects of environmental support and strategy training on older adults' use of context. Psychol. Aging 21, 499-509.

Raz, N. (2000). "Aging of the brain and its impact on cognitive performance: integration of structural and functional findings," in The Handbook of Aging and Cognition II, eds F. I. Craik and T. A. Salthouse (Mahwah, NJ: Erlbaum), 1-90.

Reimers, S., and Maylor, E.A. (2005). Task switching across the lifespan: effects of age on general and specific switch costs. Dev. Psychol. 41, 661-671.

Rogers, R. D., and Monsell, S. (1995). Costs of a predictable switch between simple cognitive tasks. J. Exp. Psychol. Gen. 124, 207-231.

Saeki, E., and Saito, S. (2004). Effect of articulatory suppression on taskswitching performance: implications for models of working memory. Memory 12, 257-271.

Verhaeghen, P., and Cerella, J. (2002). Aging, executive control, and attention: a review of meta-analyses. Neurosci. Biobehav. Rev. 26, 849-857.

Wechsler, D. (1981). The Manual for the Wechsler Adult Intelligence ScaleRevised. New York: The Psychological Corporation.

Wechsler, D. (2003). Wechsler Intelligence Scalefor Children, 4thEdn.SanAntonio, TX: Psychological Corporation.
West, R. L. (1996). An application of prefrontal cortex function theory to cognitive aging. Psychol. Bull. 120, 272-292.

Conflict of Interest Statement: The authors declare that the research was conducted in the absence of any commercial or financial relationships that could be construed as a potential conflict of interest.

Received: 25 March 2010; accepted: 06 October 2010; published online: 16 November 2010.

Citation: Kray J, Lucenet J and Blaye A (2010) Can older adults enhance task-switching performance by verbal self-instructions? The influence of working-memory load and early learning. Front. Ag. Neurosci. 2:147. doi: 10.3389/ fnagi.2010.00147

Copyright $(2010$ Kray, Lucenet and Blaye. This is an open-access article subject to an exclusive license agreement between the authors and the Frontiers Research Foundation, which permits unrestricted use, distribution, and reproduction in any medium, provided the original authors and source are credited. 